\title{
Functional testing of relapsed chronic lymphocytic leukemia guides precision medicine and maps response and resistance mechanisms. An index case
}

Relapsed chronic lymphocytic leukemia (CLL) after sequential treatment with targeted therapies has a dismal prognosis and represents an increasing unmet medical need., Immunotherapies such as chimeric antigen receptor T-cell (CAR-T) therapy or bispecific antibodies may be efficacious in this setting, but are not readily available to patients outside clinical trials. ${ }^{3}$ Direct drug testing on tumor cells can indicate treatment vulnerabilities, ${ }^{4}$ and implementation of this approach in treatment decisions for aggressive refractory hematological malignancies led to improved treatment. ${ }^{5}$ Elucidation of treatment sensitivities in multi-drug refractory CLL may thus inform novel therapeutic concepts for this patient group. Indeed, our demonstration of ex vivo sensitivity to proteasome inhibition provided the basis for the use of off-label ixazomib citrate in an index case of relapsed CLL after treatment with ibrutinib, idelalisib, alemtuzumab, and venetoclax/rituximab.

We report a high-resolution cellular and functional analysis using mass cytometry, flow cytometry, ex vivo killing assays, and drug sensitivity testing on peripheral blood mononuclear cells (PBMC) collected from the index patient at seven time points before, during, and after treatments. Our findings may indicate the molecular and cellular determinants of the treatment-responding and non-responding states of the disease and highlight the clinical value of direct drug testing to identify effective, personalized therapies for relapsed CLL.

Written informed consent was obtained before sample collection. The study was approved by the Regional Committee for Medical and Health Research Ethics of SouthEast Norway. The index patient was diagnosed with CLL at the age of 70 . The disease presented with unmutated IGVH, mutated TP53 and homozygous del(13q14). His treatment history is presented in Figure $1 \mathrm{~A}$. The patient was intolerant to ibrutinib and idelalisib, then received treatment with alemtuzumab experiencing stable disease (Figure 1A). Upon disease progression, CLL was treated with venetoclax/rituximab, and the patient obtained complete remission (CR) with undetectable minimal residual disease (UMRD) (Figure 1A). At this point, the therapy was stopped (Figure 1A). After almost 2.5 years off therapy, the disease relapsed with severe bone marrow failure. Retreatment with venetoclax failed (Figure 1A).

Serial peripheral blood samples were collected from the patient (Figure 1A). PBMC collected at T1 (after ibrutinib and idelalisib) and T6 (after venetoclax retreatment) (Figure $1 \mathrm{~A}$ ), and from three treatment-naïve CLL patients, were subjected to direct drug sensitivity screening with 93 single agents at five concentrations. ${ }^{6}$ The drug sensitivity score (DSS) was calculated based on the area under the concentration-response curve (Figure 1B). ${ }^{7}$ The drug testing confirmed statistically significant reduced drug sensitivity at $\mathrm{T} 6$ relative to $\mathrm{T} 1$ and treatment-naïve CLL ( $P<0.0001$ using 2-way ANOVA with Dunnett's multiple comparisons test). The concentration-response curves for venetoclax are shown in Figure 1C. The solid, vertical line indicates the in vitro venetoclax concentration $(2,000 \mathrm{nM})$ which corresponds to the peak plasma concentration ( 1.75 $\mu \mathrm{g} / \mathrm{mL}$ ) obtained when venetoclax is administered at 400 $\mathrm{mg} /$ day. ${ }^{8}$ As shown, venetoclax was effective at clinically achievable concentrations in PBMC collected at T1, while the sensitivity was lower in PBMC collected at T6 (Figure 1D). Indeed, the DSS was reduced with $67 \%$ at $T 6$ relative to $T 1$.

Interestingly, the CLL cells collected at T6 remained highly sensitive to the proteasome inhibitors bortezomib and ixazomib citrate (Figure $1 \mathrm{~B}$ and $\mathrm{C}$ ). Ixazomib citrate is an orally administered second-generation proteasome inhibitor approved for treatment of multiple myeloma. Preclinical effects on CLL have been observed, ${ }^{9-11}$ and phase I/II trials in non-Hodgkin lymphoma are active (www.clinicaltrials.gov). Ixazomib citrate is administered at 4 $\mathrm{mg} /$ day, which gives a maximum observed plasma concentration of $65.3 \mathrm{ng} / \mathrm{mL}^{12}$ corresponding to an in vitro concentration of about 130 nM (Figure 1C; dashed, vertical line). The patient was started on treatment with $4 \mathrm{mg}$ ixazomib citrate on day 1 of each 7-day cycle combined with $20 \mathrm{mg}$ (instead of $40 \mathrm{mg}$ to reduce side effects) dexamethasone on days 1 and 2 of each 7-day cycle. This is according to the summary of product characteristics (SPC) for ixazomib citrate and the approved dosing according to the European Medicines Agency. The patient was transfusiondependent with very severe thrombocytopenia $(<10 \times 10 \% / \mathrm{L}$, Figure 1D) which made an oral proteasome inhibitor preferable. Therapy resulted in increased numbers of reticulocytes, thrombocytes, and hemoglobin, indicating that the therapy was effective (Figure 1D). The bone marrow response to treatment (T7) is illustrated in Figure 1E. At present, $>120$ days after treatment initiation, the patient is transfusion-independent with no bleedings, he is able to exercise and has an active life. 
A $\sigma^{M}$ UM-CLL

TP53mut, del(13q14)

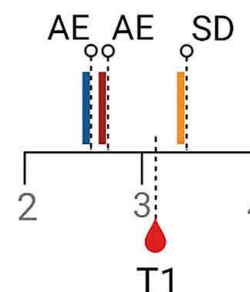

Ibrutinib

Idelalisib

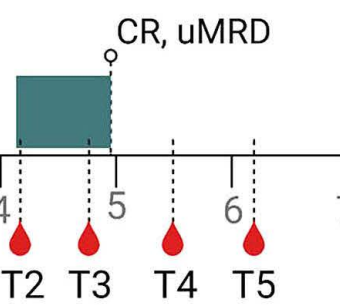

Alemtuzumab

Venetoclax (+ Rituximab)
C

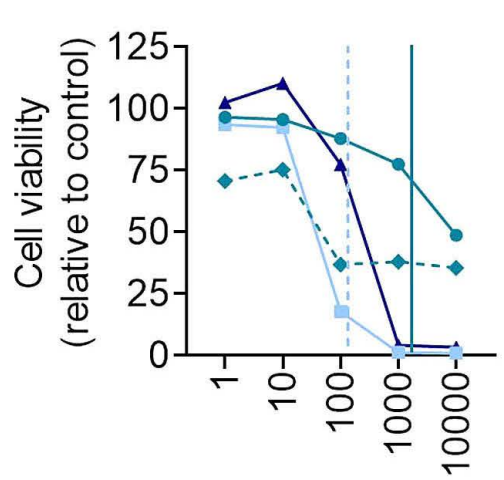

Drug concentration (nM)

D

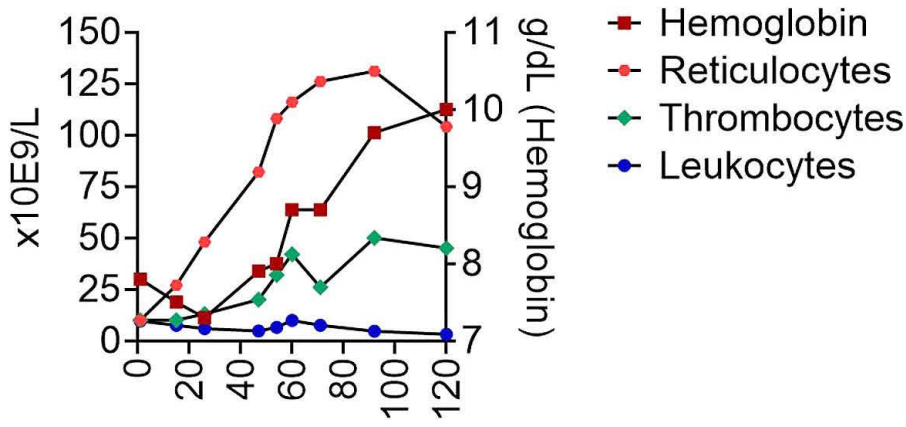

Days since start of Ixazomib Citrate

E

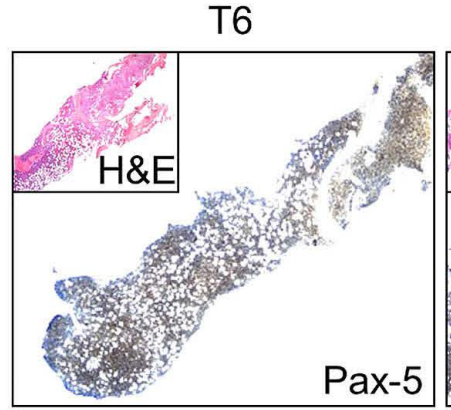

$\rightarrow$ Venetoclax (T1)

$\rightarrow$ Venetoclax (T6)

- Ixazomib Citrate (T6)

- Bortezomib (T6)
B

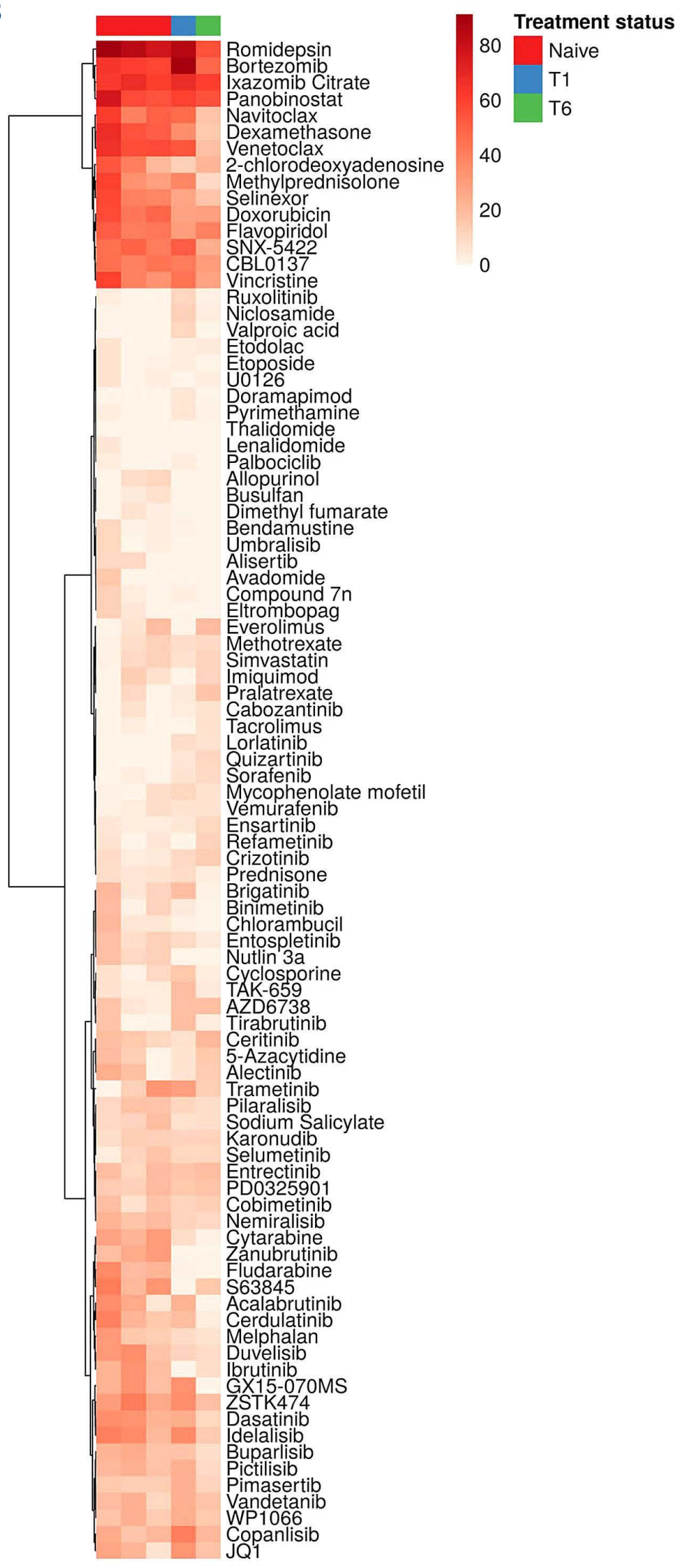

Figure 1. Ixazomib citrate is effective against chronic lymphocytic leukemia ex vivo and in vivo. (A) Graphical illustration of the index patient's treatment history, shown as years since diagnosis. Peripheral blood mononuclear cells (PBMC) were collected at the indicated time points T1-T7. The figure was created with BioRender.com. (B) PBMC collected from 3 treatment-naïve chronic lymphocytic leukemia (CLL) patients, as well as at T1 and T6 from the index patient, were co-cultured with $\mathrm{CD}^{2} 0 \mathrm{~L}^{+}$, BAFF $\mathrm{B}^{+}$, and APRIL $^{+} L$ cells (ratio 1:1:1) for 24 hours (h) prior to initiation of the experiment to mimic the tumor microenvironment. The $L$ cells were then removed and the CLL cells were treated with the indicated 93 single agents at 5 different concentrations (1-10,000 $\mathrm{nM}$ ) for $72 \mathrm{~h}$. Cell viability was assessed with the CellTiter-Glo luminescent assay. The response readouts were normalized to the negative ( $0.1 \% \mathrm{DMSO})$ and positive $(100 \mu \mathrm{M}$ benzethonium chloride) controls. The heatmap was created using ClustVis (https://biit.cs.us.ee/clustvis/) and illustrates the calculated DSS on a scale from 0-100 (see key, right). Rows are clustered using Manhattan distance and Ward (unsquared distances) linkage. (C) Relative cell viability of PBMC collected at T1 or T6 in response to venetoclax, bortezomib or ixazomib citrate exposure. The experiment is described in (B). The solid, vertical line indicates the maximum plasma concentration of venetoclax reported for patients treated with $400 \mathrm{mg} /$ day. The dashed, vertical line indicates the maximum plasma concentration of ixazomib citrate for patients treated with $4 \mathrm{mg} / \mathrm{day}$. (D) Blood counts of the index patient in response to treatment with ixazomib citrate + dexamethasone. (E) Anti-Pax-5- and hematoxylin and eosin (H\&E)-stained sections of bone marrow from T6 and T7, at 2x magnification. Pax-5-positive cells are shown in brown. CLL cells were reduced from 90\% (T6) to $65 \%$ (T7) of the bone marrow cellularity. AE: adverse event; CR: complete remission; NR: no response; SD: stable disease; UM-CLL: IGVH unmutated CLL; UMRD: undetectable minimal residual disease. 
In order to map the cellular responses to venetoclax treatment, PBMC collected at T1-T3 (Figure 1A) were subjected to immune cell phenotyping by single-cell mass cytometry (Figure $2 \mathrm{~A}$ ). As expected, the $\operatorname{lgM}^{+} \mathrm{CD} 19^{+} \mathrm{CLL} B-$ cell population dominated at T1-T2 (Figure 2A). However, after 7 months on venetoclax, this population was almost eradicated (T3, Figure $2 A)$. This demonstrated the efficacy of venetoclax and aligned with the subsequently achieved $C R$ with UMRD (Figure 1A). Interestingly, as a result of treatment, the $\mathrm{CD}^{+} \mathrm{T}$-cell and $\mathrm{CD}^{+} 4^{+}$monocyte populations were restored (Figure 2A). Reshaping of the immune cell composition in response to venetoclax treatment has been reported..$^{13}$

Of particular interest, we also observed that the CD56 ${ }^{+}$ natural killer (NK) cell population had significantly expanded at T3 (41\% of PBMC at T3 vs. $0.4 \%$ at T1, Figure $2 \mathrm{~A}$ ). This population included the standard CD56 ${ }^{\text {dim }} C D 16^{\text {hi }}$ cells and expanded, immature CD56 $6^{\text {bright }} \mathrm{CD} 16^{\text {low }}$ cells. Both cell types were similarly activated (HLA-DR ${ }^{+}, \mathrm{Ki} 67^{+}$, Figure 2B). NK cell numbers continued to increase after venetoclax therapy was held, and were still high (>20\%) more than 1 year prior to disease relapse (T5, Figure $2 \mathrm{C}$ ), but had fallen below $10 \%$ when the disease was progressing (T6, Figure 2C). The expanded NK cells (T3-T5) contained a large fraction of CD56 bright cells (34-54\%) (Figure 2D), possibly reflecting immature cells recruited from the bone marrow. We next investigated the lytic activity of purified, bulk NK cells at T4-T7 against autologous CLL or K562 cells. NK
A

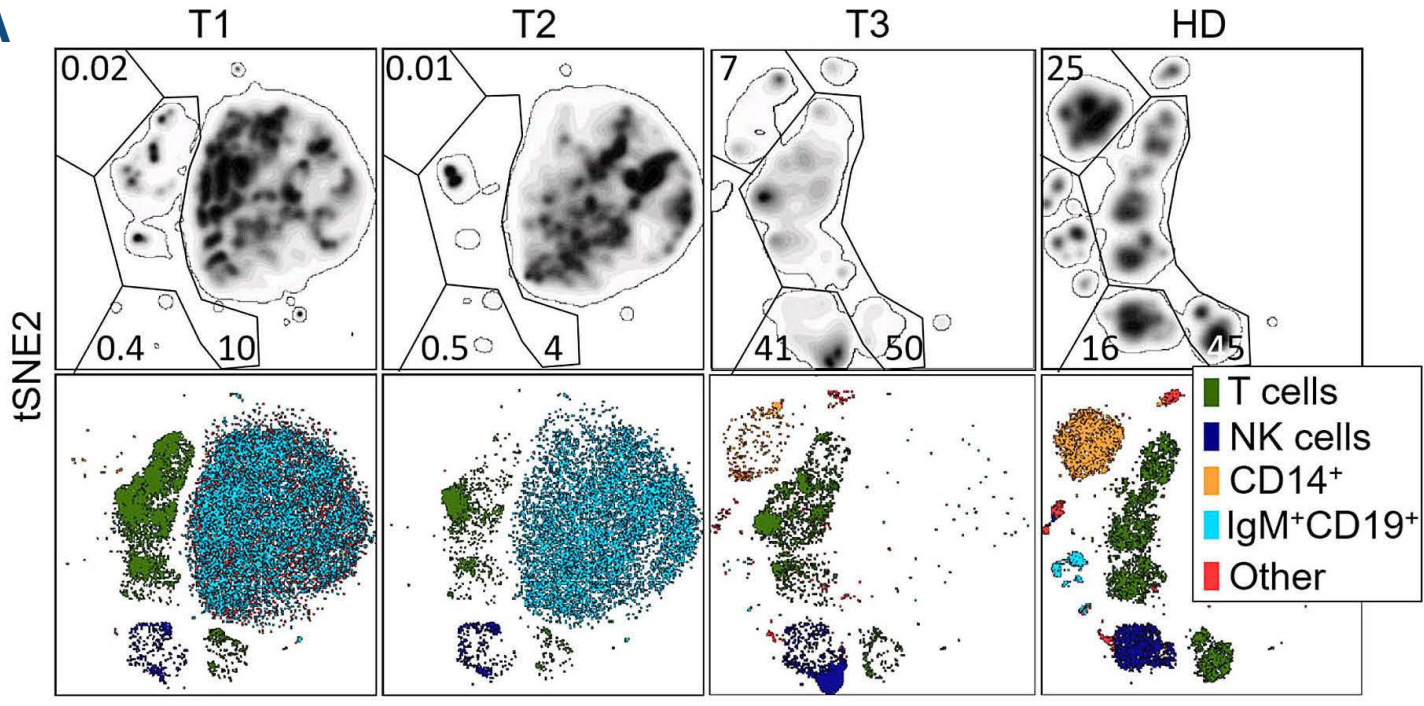

tSNE1
B

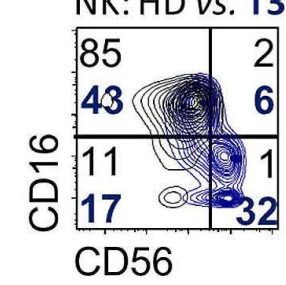

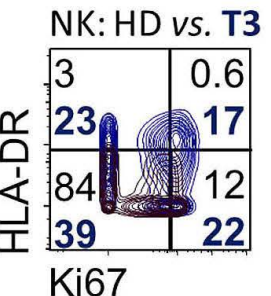

C

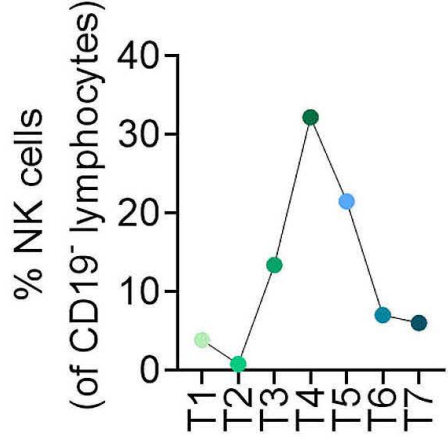

D

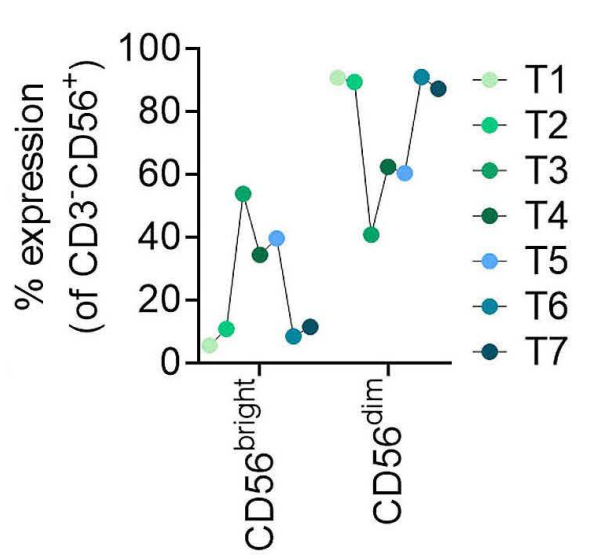

E

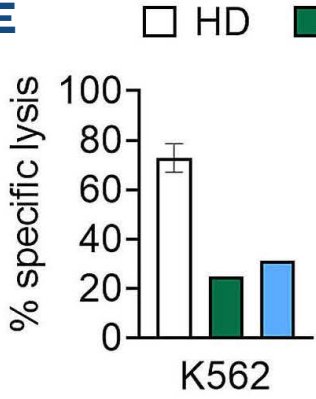

T4 $\square \mathrm{T} 5$
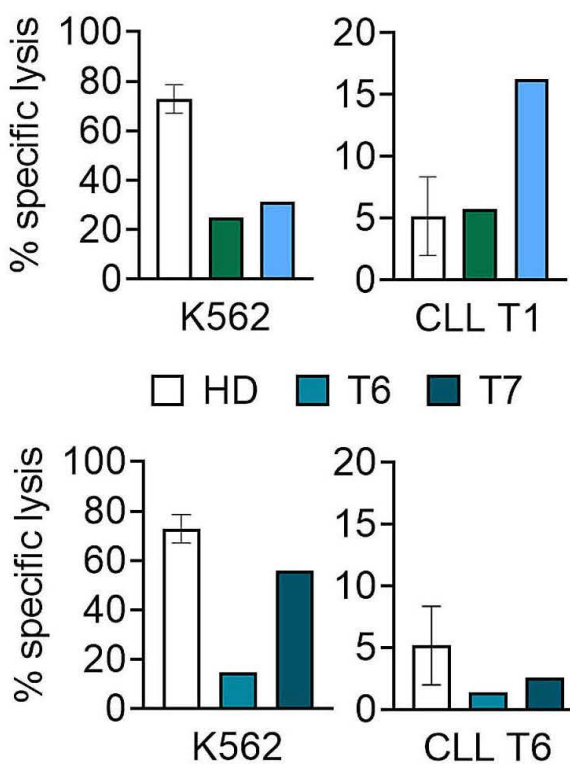
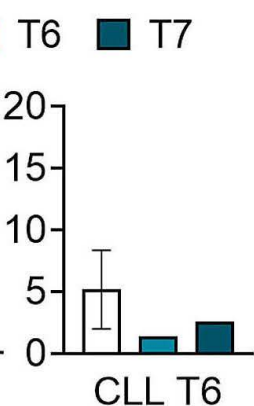

$\mathbf{F}$

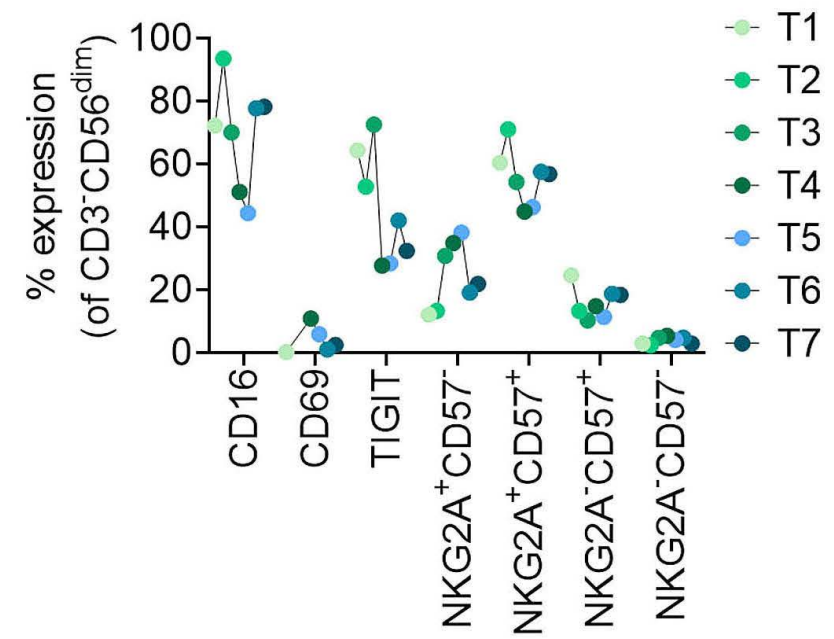

Figure 2. Evolution of natural killer cells in response to venetoclax and ixazomib citrate treatments. (A) Opt-SNE representation of immune cells (CyTOF) based on protein expression of lineage-defining surface markers in peripheral blood mononuclear cells (PBMC) collected at T1-T3 and from a healthy age-matched blood donor (HD). The top row shows density of cells. The numbers indicate percentage of cells in the respective gate. The bottom row shows back-gated chronic lymphocytic leukemia (CLL) cells and naïve $\mathrm{B}$ cells $\left(\mathrm{IgM}^{+} \mathrm{CD} 19^{+}\right)$, T cells $\left(\mathrm{CD} 3^{+}\right.$, including natural killer $[\mathrm{NK}] \mathrm{T}$ cells $\mathrm{CD} 3^{+} \mathrm{CD} 56^{+}$, lower regions), monocytes $\left(\mathrm{CD} 14^{+}\right)$, and NK cells (CD56+) (see color key). (B) Gated NK cells shown in overlayed contour plots for a healthy age-matched blood donor (HD; black) and CLL T3 (blue). CD56 vs. CD16 (upper panel) and Ki67 vs. HLA-DR (lower panel) are shown. Quadrant percentages are shown in black (HD) and blue (T3) font. (C) Frequencies of CD3-CD56 ${ }^{+} \mathrm{NK}^{-}$cells among CD19- lymphocytes at T1-T7, detected by flow cytometry. (D) Frequencies of CD56 bright and CD56 dim NK cell subsets within CD3-CD56 ${ }^{+}$NK cells at T1-T7, detected by flow cytometry. (E) Specific lysis of K562 cells or CLL blasts (from T1 or T6) by CD56+-positively selected NK cells isolated at indicated time points or from a healthy donor (HD). Cell death was monitored as $\mathrm{Pl}^{+}$events within CFSE prestained CLL blasts. (F) Frequencies of indicated subsets within CD3-CD56 dim NK cells at T1-T7, detected by flow cytometry. 
cells from T5 exerted increased cytotoxicity against the autologous T1 CLL cells, relative to NK cells from T4 (Figure $2 \mathrm{E}$ ). Killing of K562 cells was lowest at T6, suggesting reversal of the augmented NK cell-mediated cytotoxicity at relapse (Figure 2E). Cytotoxicity was higher at T7, but no activity against T6 CLL cells was observed (Figure 2E). Further analysis showed a temporal decreased expression of CD16 and an increased expression of the activation marker CD69 on CD56 dim NK cells between T3-T5 (Figure $2 \mathrm{~F})$. This matched the expression of the exhaustion marker TIGIT that initially was high, then normalized, except for a temporal increase at T6 (Figure 2F). Further, a compensatory temporal increase in the less mature NKG2A ${ }^{+}$CD57- subset was observed after venetoclax treatment (T3-T5), with concomitant reduction of terminally differentiated $\mathrm{CD} 57^{+} \mathrm{NK}$ cells (Figure 2F). Collectively, these data show an activated NK cell compartment with signs of exhaustion and enhanced killing efficacy after venetoclax treatment $(>T 3)$ with normalization at relapse (T6) in this patient.

In order to further evaluate the mechanism of venetoclax response and resistance in the index patient and of ixazomib citrate response, we next profiled the expression and activation status of 30 intracellular proteins in the serial CLL samples ${ }^{14}$ (Figure 3A). Interestingly, PBMC col- lected when the patient was responding to either venetoclax or ixazomib citrate ( $\mathrm{T} 4$ and $\mathrm{T} 7$, respectively), showed a more similar profile than PBMC collected when the patient had active disease (T1 and T6; Figure $3 \mathrm{~A}$ ). Notably, expression of BCL-2 was significantly lower at $\mathrm{T} 4$ than at the other time points (Figure 3B), while Bim dropped at T6 (Figure 3B). Proteins downstream of the Bcell receptor, including BTK, MEK1, and S6-ribosomal protein, displayed enhanced phosphorylation levels at time of relapse (T6 vs. T4; Figure 3A and B). Upregulation of the MEK pathway in combination with decreased Bim has also previously been associated with drug resistance in CLL. ${ }^{15}$ In general, treatment with ixazomib citrate restored the protein expression and activation levels to a similar level as when the patient was in remission after venetoclax treatment (T7 vs. T4; Figure 3B).

Taken together, our study provides mechanistic insight to clinical response and resistance to targeted therapies (Figure $3 \mathrm{C}$ ), as well as proof-of-concept for direct drug testing as a method to guide effective personalized therapy for relapsed CLL. Since drug sensitivity screens can be performed and analyzed in only 5 days, it is possible that this method can be used as a companion diagnostic for CLL patients in need of therapy. Clinical trials are needed to test this approach to functional precision medicine.

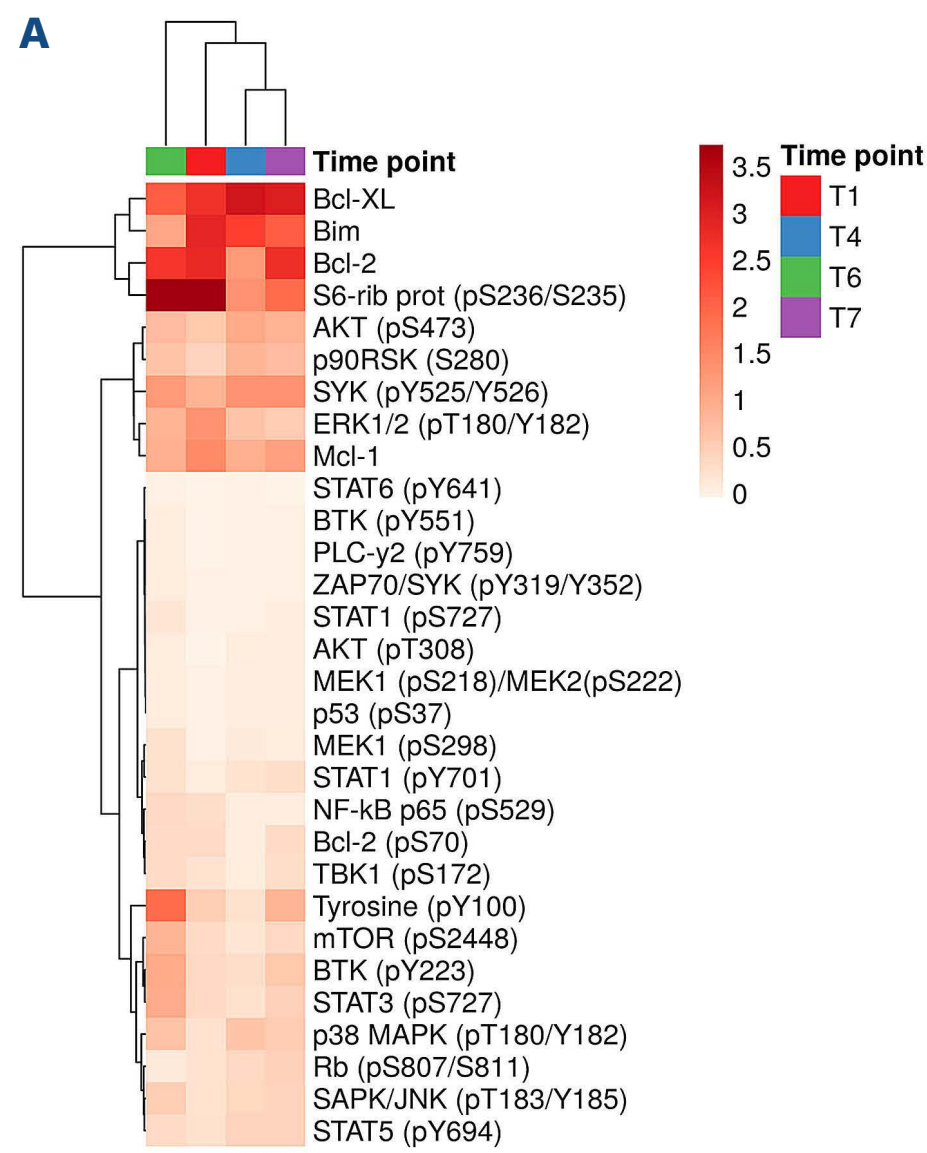

B

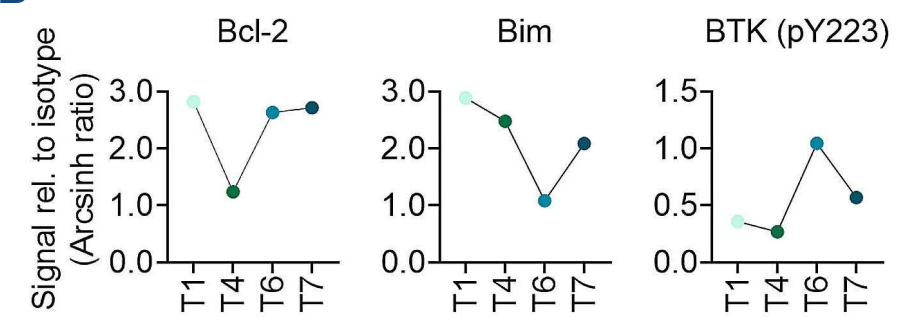

C

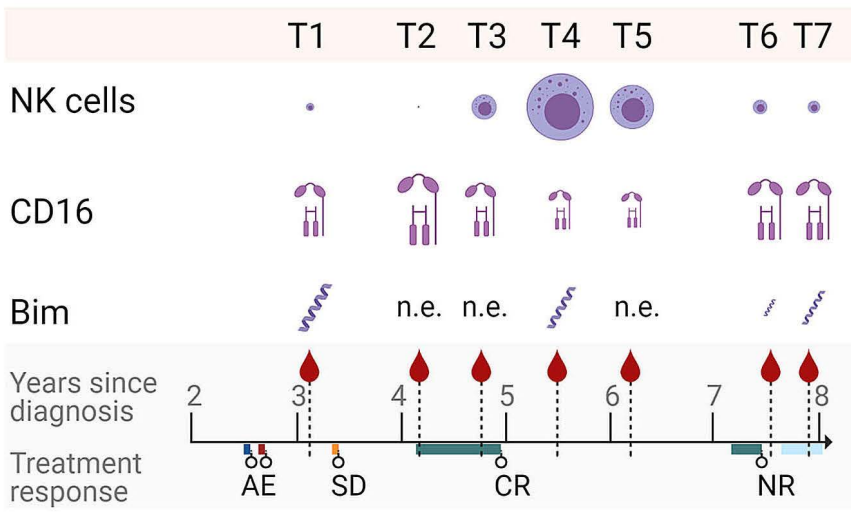

Figure 3. Evolution of protein profile in response to venetoclax and ixazomib citrate treatments. (A) Peripheral blood mononuclear cells (PBMC) collected at T1, T4, T6, and T7 were fixed, permeabilized and stained with the indicated antibodies. Signals in $\mathrm{CD}_{19}{ }^{+}$cells were analyzed by flow cytometry. Raw data were analyzed in Cytobank (https:/cellmass.cytobank.org/cytobank/) and transformed to an arcsinh ratio relative to the signal in isotype control-stained cells, which was set to zero. The heatmap was created using ClustVis (https://biit.cs.us.ee/clustvis/). Both rows and columns are clustered using Manhattan distance and Ward linkage. (B) Protein expression and phosphorylation levels detected in (A). (C) Graphical summary of some of the cellular characteristics of the PBMC collected at T1-T7. The size of the symbols reflects the relative detected level at each sampling time. Refer to Figure 1A for details regarding treatment history. n.e: not established. The figure was created with BioRender.com. 


\section{Authors}

Sigrid S. Skånland, ${ }^{1,2}$ Marit Inngjerdingen, ${ }^{3}$ Henrik Bendiksen, $, 1,2$ Jamie York, ${ }^{2,4}$ Signe Spetalen, ${ }^{5}$ Ludvig A. Munthe ${ }^{2,4}$ and Geir E. Tjønnfjord ${ }^{2,6}$

1Department of Cancer Immunology, Institute for Cancer Research, Oslo University Hospital; ${ }^{2} \mathrm{~K}$. G. Jebsen Center for B-Cell Malignancies, Institute of Clinical Medicine, University of Oslo; ${ }^{3}$ Department of Pharmacology, Institute of Clinical Medicine, University of Oslo; ${ }^{4}$ Department of Immunology, Oslo University Hospital; ${ }^{5}$ Department of Pathology, Oslo University Hospital and ${ }^{6}$ Department of Hematology, Oslo University Hospital, Oslo, Norway

Correspondence:

S.S. SKÅNLAND - sigrid.skanland@ous-research.no

https://doi.org/10.3324/haematol.2021.280393

Received: November 22, 2021.

Accepted: February 23, 2022.

Prepublished: March 3, 2022.

(2)22 Ferrata Storti Foundation

Haematologica material is published under a CC-BY-NC license @@

\section{Disclosures}

No conflicts of interest to disclose.

\section{Contributions}

SSS designed the research with MI and LAM; SSS, MI, HB and JY performed experiments and analyzed data with LAM; SS performed bone marrow histopathology; GET contributed with patient samples and provided clinical care; SSS wrote the manuscript. All authors read and commented on draft versions of the manuscript and approved the final version.

\section{Acknowledgements}

We are thankful to all study participants. We thank the High Throughput Biomedicine Unit at Institute for Molecular Medicine Finland (FIMM) for assistance with drug sensitivity screens.

\section{Funding}

The work was supported by the Research Council of Norway under the frame of ERA PerMed (to SSS, project number 322898), Lilly Constance og Karl Ingolf Larssons stiftelse (to SSS), and Stiftelsen Kristian Gerhard Jebsen (to GET and LAM, grant 19).

\section{Data-sharing statement}

The data that support the findings of this study are available from the corresponding author (sigrid.skanland@ous-research.no) upon reasonable request.

\section{References}

1. Lew TE, Lin VS, Cliff ER, et al. Outcomes of patients with CLL sequentially resistant to both BCL2 and BTK inhibition. Blood Adv. 2021;5(20):4054-4058.

2. Mato AR, Davids MS, Sharman J, et al. Recognizing unmet need in the era of targeted therapy for CLL/SLL: "What's past is prologue" (Shakespeare). Clin Cancer Res.

2022 Feb 15; 28(4):603-608.

3. Skånland SS, Mato AR. Overcoming resistance to targeted therapies in chronic lymphocytic leukemia. Blood Adv. 2021;5(1):334-343.

4. Letai A, Bhola P, Welm AL. Functional precision oncology: testing tumors with drugs to identify vulnerabilities and novel combinations. Cancer Cell. 2022;40(1):26-35.

5. Kornauth C, Pemovska T, Vladimer GI, et al. Functional precision medicine provides clinical benefit in advanced aggressive hematologic cancers and identifies exceptional responders. Cancer Discov. 2022;12(2):372-387.

6. Skånland SS, Cremaschi $\mathrm{A}$, Bendiksen $\mathrm{H}$, et al. An in vitro assay for biomarker discovery and dose prediction applied to ibrutinib plus venetoclax treatment of CLL. Leukemia. 2020;34(2):478-487.

7. Yadav B, Pemovska T, Szwajda A, et al. Quantitative scoring of differential drug sensitivity for individually optimized anticancer therapies. Sci Rep. 2014;4:5193.

8. Salem AH, Agarwal SK, Dunbar M, et al. Pharmacokinetics of venetoclax, a novel BCL-2 inhibitor, in patients with relapsed or refractory chronic lymphocytic leukemia or non-Hodgkin lymphoma. J Clin Pharmacol. 2017;57(4):484-492.

9. Duechler M, Shehata M, Schwarzmeier JD, et al. Induction of apoptosis by proteasome inhibitors in B-CLL cells is associated with downregulation of CD23 and inactivation of Notch2. Leukemia. 2005;19(2):260-267.

10. Ruiz S, Krupnik Y, Keating $M$, et al. The proteasome inhibitor $\mathrm{NPI}-0052$ is a more effective inducer of apoptosis than bortezomib in lymphocytes from patients with chronic lymphocytic leukemia. Mol Cancer Ther. 2006;5(7):1836-1843.

11. Billard C. Apoptosis inducers in chronic lymphocytic leukemia. Oncotarget. 2014;5(2):309-325.

12. Suzuki K, Handa H, Chou T, et al. Phase 1 study of ixazomib alone or combined with lenalidomide-dexamethasone in Japanese patients with relapsed/refractory multiple myeloma. Int J Hematol. 2017;105(4):445-452.

13. de Weerdt I, Hofland T, de Boer R, et al. Distinct immune composition in lymph node and peripheral blood of CLL patients is reshaped during venetoclax treatment. Blood Adv. 2019;3(17):2642-2652.

14. Skånland SS. Phospho flow cytometry with fluorescent cell barcoding for single cell signaling analysis and biomarker discovery. J Vis Exp. 2018;(140):e58386.

15. Hallaert DY, Jaspers A, van Noesel CJ, et al. c-Abl kinase inhibitors overcome CD40-mediated drug resistance in CLL: implications for therapeutic targeting of chemoresistant niches. Blood. 2008;112(13):5141-5149. 